\title{
REGULARITY AND FAST ESCAPING POINTS OF ENTIRE FUNCTIONS
}

\author{
P. J. RIPPON AND G. M. STALLARD
}

\begin{abstract}
Let $f$ be a transcendental entire function. The fast escaping set $A(f)$, various regularity conditions on the growth of the maximum modulus of $f$, and also, more recently, the quite fast escaping set $Q(f)$ have all been used to make progress on fundamental questions concerning the iteration of $f$. In this paper we establish new relationships between these three concepts.

We prove that a certain weak regularity condition is necessary and sufficient for $Q(f)=A(f)$ and give examples of functions for which $Q(f) \neq A(f)$.

We also apply a result of Beurling that relates the size of the minimum modulus of $f$ to the growth of its maximum modulus in order to establish that a stronger regularity condition called log-regularity holds for a large class of functions, in particular for functions in the Eremenko-Lyubich class $\mathcal{B}$.
\end{abstract}

\section{INTRODUCTION}

Let $f: \mathbb{C} \rightarrow \mathbb{C}$ be a transcendental entire function and denote by $f^{n}, n=$ $0,1,2, \ldots$, the $n$th iterate of $f$. The Fatou set $F(f)$ is the set of points $z \in \mathbb{C}$ such that $\left(f^{n}\right)_{n \in \mathbb{N}}$ forms a normal family in some neighborhood of $z$. The complement of $F(f)$ is called the Julia set $J(f)$ of $f$. An introduction to the properties of these sets can be found in [2].

In recent years there has been much interest in understanding the structure of the escaping set $I(f)$ of $f$, defined as follows:

$$
I(f)=\left\{z: f^{n}(z) \rightarrow \infty \text { as } n \rightarrow \infty\right\} .
$$

The first general results about $I(f)$ were obtained by Eremenko in [9], who showed that $I(f) \cap J(f) \neq \emptyset$ and $J(f)=\partial I(f)$, and also that all the components of $\overline{I(f)}$ are unbounded. Much of this work has been directed towards solving what is known as Eremenko's conjecture, which states that all the components of $I(f)$ itself are unbounded. Though this conjecture is still unsolved, much progress has been made in special cases (see [20] for example), and in the general case it is known [14] that $I(f)$ has at least one unbounded component.

This result in the general case was proved by using the fast escaping set $A(f)$, which consists of points whose iterates tend to $\infty$ as fast as possible. The set $A(f)$ was introduced in [4] and can be defined as follows; see [16]. Let

$$
M(r)=M(r, f)=\max _{|z|=r}|f(z)|, \quad r>0,
$$

and put

$$
A(f)=\left\{z: \text { there exists } \ell \in \mathbb{N} \text { such that }\left|f^{n+\ell}(z)\right| \geq M^{n}(R) \text {, for } n \in \mathbb{N}\right\},
$$

2010 Mathematics Subject Classification. Primary 37F10, Secondary 30D05.

Both authors were supported by the EPSRC grant EP/H006591/1. 
where $M^{n}(r)$ denotes iteration of $M(r)$, and $R>0$ is any value such that $M(r)>$ $r$ for $r \geq R$. The set $A(f)$ has many strong properties that can be used in the study of $I(f)$ and $J(f)$; see [16]. For example, all the components of $A(f)$ are unbounded.

In 16] we gave a weaker condition on the iterates of a point that is sufficient to ensure that the point is in $A(f)$. To be precise we showed that if $\mu(r)=\varepsilon M(r)$, $r>0$, where $\varepsilon \in(0,1)$, then

(1.1) $A(f)=\left\{z\right.$ : there exists $\ell \in \mathbb{N}$ such that $\left|f^{n+\ell}(z)\right| \geq \mu^{n}(R)$, for $\left.n \in \mathbb{N}\right\}$,

where $R>0$ is sufficiently large to ensure that $\mu(r)>r$, for $r \geq R$.

Sixsmith [22, Theorem 2] recently gave a weaker but somewhat more involved condition than the one in (1.1) which ensures that points are in $A(f)$.

A further weakening of (1.1) is to replace $\varepsilon M(r)$ by $M(r)^{\varepsilon}$. In other words, for $0<\varepsilon<1$, we let

$$
\mu_{\varepsilon}(r)=M(r)^{\varepsilon}, \quad r>0
$$

and consider the set

$$
Q_{\varepsilon}(f)=\left\{z: \text { there exists } \ell \in \mathbb{N} \text { such that }\left|f^{n+\ell}(z)\right| \geq \mu_{\varepsilon}^{n}(R) \text {, for } n \in \mathbb{N}\right\},
$$

where $R>0$ has the property that $\mu_{\varepsilon}(r)>r$ for $r \geq R$. It is easy to check that the definition of $Q_{\varepsilon}(f)$ is independent of the choice of $R$ with the property that $\mu_{\varepsilon}(r)>r$ for $r \geq R$.

Points that lie in $Q_{\varepsilon}(f)$ for some $\varepsilon, 0<\varepsilon<1$, have played a significant role in recent work related to both Eremenko's conjecture and a conjecture of Baker that all transcendental entire functions of sufficiently small order have no unbounded Fatou components (see [17]), and also in papers related to the Hausdorff measure and Hausdorff dimension of various Julia and escaping sets (see the remark following Theorem 1.1). Because of the increased significance of these points we define the set

$$
Q(f)=\bigcup_{0<\varepsilon<1} Q_{\varepsilon}(f)
$$

to be the quite fast escaping set of $f$. It follows immediately from these definitions that

$$
A(f) \subset Q_{\varepsilon}(f) \subset Q(f) \subset I(f), \text { for } 0<\varepsilon<1 \text {. }
$$

The central question in this paper concerns the relationship between $Q(f)$ and $A(f)$, and we show that for many transcendental entire functions $Q(f)$ is identical to $A(f)$. However, we also give examples of entire functions for which $Q(f) \neq A(f)$; further such examples, which are related to Eremenko's conjecture and Baker's conjecture and are much more complicated, are given in [18].

An important special case of our results is the following theorem concerning the much-studied Eremenko-Lyubich class $\mathcal{B}$, which consists of transcendental entire functions whose set of singular values (that is, critical values and asymptotic values) is bounded.

Theorem 1.1. Let $f$ be a transcendental entire function in the class $\mathcal{B}$. Then $Q(f)=A(f)$. 
Remark. Theorem 1.1 implies that some results concerning the Hausdorff measure and Hausdorff dimension of the escaping sets and Julia sets of certain functions in the class $\mathcal{B}$ also hold for the fast escaping sets of such functions. In particular, [5, Theorem 1.1] and [13, Theorem 1.1] were proved by considering points in $Q(f)$ (although this is not stated explicitly) and so, since the functions in those results are in the class $\mathcal{B}$, it follows from Theorem 1.1 that the conclusions of those results also hold for $A(f)$.

The proof of Theorem 1.1 is in two steps. First we introduce a regularity condition on $f$ which implies that $Q(f)=A(f)$. Let $R>0$ be any value such that $M(r)>r$ for $r \geq R$. We say that $f$ is

- $\varepsilon$-regular, where $0<\varepsilon<1$, if there exists $r=r(R)>0$ such that

$$
\mu_{\varepsilon}^{n}(r) \geq M^{n}(R), \text { for } n \in \mathbb{N},
$$

or, equivalently, if there exists $\ell=\ell(R) \in \mathbb{N}$ such that

$$
\mu_{\varepsilon}^{n+\ell}(R) \geq M^{n}(R), \text { for } n \in \mathbb{N} ;
$$

- weakly regular if $f$ is $\varepsilon$-regular for all $0<\varepsilon<1$.

We note that the definition of $\varepsilon$-regularity is independent of the value of $R$ with the property that $M(r)>r$ for $r \geq R$.

Roughly speaking, the aim of these regularity conditions, and other related conditions to be introduced later in the paper, is to prevent the maximum modulus of a transcendental entire function from behaving on long intervals in a way that is too similar to that of a polynomial.

We show that, not only is weak regularity a sufficient condition for $Q(f)=$ $A(f)$, but it is also a necessary condition for $Q(f)=A(f)$.

Theorem 1.2. Let $f$ be a transcendental entire function and $0<\varepsilon<1$. Then

(a) $f$ is $\varepsilon$-regular if and only if $Q_{\varepsilon}(f)=A(f)$;

(b) $f$ is weakly regular if and only if $Q(f)=A(f)$.

The proof that weak regularity is a sufficient condition for $Q(f)=A(f)$ is straightforward. However, in order to prove that it is also a necessary condition we require a rather general result from [19] that guarantees the existence of points with a prescribed rate of escape; see Section 3 .

The second (and main) step in the proof of Theorem[1.1 is to show that if $f$ is in the class $\mathcal{B}$, then $f$ is weakly regular. Recall that if $f$ is in the class $\mathcal{B}$, then $f$ is bounded on some path to $\infty$; see [10, page 993]. Our next result shows that $f$ is weakly regular whenever $f$ is bounded on a path to $\infty$ and indeed under a much weaker hypothesis involving the minimum modulus of $f$, which is defined as follows:

$$
m(r)=m(r, f)=\min _{|z|=r}|f(z)|, \quad r>0
$$

Thus Theorem 1.1 can be viewed as a special case of the following result.

Theorem 1.3. Let $f$ be a transcendental entire function such that, for some $r(f)>1$,

$$
m(r) \leq M(r)^{1-K / \log r}, \text { for } r \geq r(f),
$$

where $K=4 \log 4$. Then $f$ is weakly regular, and hence $Q(f)=A(f)$. 
In fact we show that (1.4) implies that $f$ satisfies a stronger regularity condition called 'log-regularity' that we define in Section 4. This log-regularity condition was introduced by Anderson and Hinkkanen in [1] in connection with Baker's conjecture, and it is easier to check than weak regularity. We prove Theorem 1.3 by using a comparatively unknown result of Beurling from his thesis [7], which relates the size of the minimum modulus of a function to the growth of its maximum modulus.

The structure of the paper is as follows. We begin in Section 2 by discussing some of the basic properties of $Q(f)$. In Section 3 we prove Theorem 1.2 and also construct functions for which $Q(f) \neq A(f)$.

In Section 4, we discuss several regularity conditions for a transcendental entire function and the relationships between them; in particular, we show that log-regularity implies weak regularity and that $\varepsilon$-regularity is equivalent to a type of regularity introduced in [16]. Then in Section 5 we prove Theorem 1.3 . Finally, in Section 6 we construct examples in order to show that $\varepsilon$-regularity, weak-regularity and log-regularity are not equivalent conditions.

\section{BASIC PROPERTIES OF $Q(f)$}

In this section we show that $Q(f)$ has many of the same basic properties as $I(f)$ and $A(f)$. As mentioned earlier, Eremenko [9] established the following properties of $I(f)$ :

$$
I(f) \neq \emptyset, \quad I(f) \cap J(f) \neq \emptyset, \quad J(f)=\partial I(f),
$$

and he also showed that $\overline{I(f)}$ has no bounded components. The fast escaping set also has the properties listed in (2.1), and in addition $A(f)$ itself has no bounded components, as noted earlier; see [4] and [14].

The sets $Q(f)$ and $Q_{\varepsilon}(f)$ have similar properties to $I(f)$. The proofs of these properties are similar to those for $I(f)$ so we give only brief details for $Q(f)$.

Theorem 2.1. Let $f$ be a transcendental entire function. Then

$$
Q(f) \neq \emptyset, \quad Q(f) \cap J(f) \neq \emptyset, \quad J(f)=\overline{Q(f) \cap J(f)}, \quad J(f)=\partial Q(f),
$$

and $\overline{Q(f)}$ has no bounded components. Similar properties hold for each set $Q_{\varepsilon}(f)$, where $0<\varepsilon<1$.

Proof. The first three properties in (2.2) are immediate since these properties hold for $A(f)$ (see [16, Theorem 5.1]) and $A(f) \subset Q(f)$.

Because $Q(f)$ is infinite and completely invariant under $f$, we have $J(f) \subset$ $\overline{Q(f)}$, and this implies that $J(f) \subset \partial Q(f)$ since any open subset of $Q(f)$ is contained in $F(f)$. Also, no point of $\partial Q(f)$ lies in $F(f)$ since any such point would have a neighbourhood in $Q(f)$ by an application of the distortion theorem for iterates in Fatou components; see [2, Lemma 7]. Hence $J(f)=\partial Q(f)$.

Finally, if $\overline{Q(f)}$ has a bounded component, $E$ say, then there is an open topological annulus $A$ lying in the complement of $\overline{Q(f)}$ that surrounds $E$. Since $\overline{Q(f)}$ is completely invariant under $f$, we deduce by Montel's theorem that $A \subset$ $F(f)$ and since $J(f)=\partial Q(f)$ we deduce that $A$ is contained in a multiply connected Fatou component. But any multiply connected Fatou component of $f$ 
is contained in $A(f)$ (see [14, Theorem 2] or [16, Theorem 4.4]) and hence in $Q(f)$, so we obtain a contradiction. This completes the proof of Theorem 2.1 .

Remark In view of the considerable interest in Eremenko's conjecture, it is natural to ask whether all the components of $Q(f)$ are unbounded.

\section{Proof of Theorem 1.2}

In this section we prove Theorem 1.2 and also construct examples for which $Q(f) \neq A(f)$. The proof of Theorem 1.2 uses the following general result from [19, Theorem 1.4].

Theorem 3.1. Let $f$ be a transcendental entire function. There exists $R=$ $R(f)>0$ with the property that whenever $\left(a_{n}\right)$ is a positive sequence such that

$$
a_{n} \geq R \text { and } a_{n+1} \leq M\left(a_{n}\right), \text { for } n \in \mathbb{N},
$$

there exists a point $\zeta \in J(f)$ and a sequence $\left(n_{j}\right)$ with $n_{j} \rightarrow \infty$ as $j \rightarrow \infty$, such that

$$
\left|f^{n}(\zeta)\right| \geq a_{n}, \text { for } n \in \mathbb{N}, \text { but }\left|f^{n_{j}}(\zeta)\right| \leq M^{2}\left(a_{n_{j}}\right) \text {, for } j \in \mathbb{N} \text {. }
$$

This result guarantees in particular that for any given rate of escape that is at most that of 'fast escape' there exist points that escape at least at this given rate and in some sense at no faster rate.

Proof of Theorem 1.2. We first prove part (a), which states that $f$ is $\varepsilon$-regular if and only if $Q_{\varepsilon}(f)=A(f)$. Let $0<\varepsilon<1$ and let $R>0$ be so large that $\mu_{\varepsilon}(r)>r$ for $r \geq R$ and so that Theorem 3.1 can be applied with this value of $R$.

Suppose first that $f$ is $\varepsilon$-regular. If $z \in Q_{\varepsilon}(f)$, then there exists $\ell \in \mathbb{N}$ such that

$$
\left|f^{n+\ell}(z)\right| \geq \mu_{\varepsilon}^{n}(R), \text { for } n \in \mathbb{N} .
$$

Let $r=r(R)$ be as in the definition of $\varepsilon$-regularity. Then there exists $m \in N$ such that $\mu_{\varepsilon}^{m}(R)>r$ so

$$
\left|f^{n+\ell+m}(z)\right| \geq \mu_{\varepsilon}^{n+m}(R) \geq \mu_{\varepsilon}^{n}(r) \geq M^{n}(R), \text { for } n \in \mathbb{N},
$$

and hence $z \in A(f)$. Thus $Q_{\varepsilon}(f)=A(f)$.

The proof of part (a) in the opposite direction uses Theorem 3.1. The idea is to show that if the function $f$ is not $\varepsilon$-regular, where $0<\varepsilon<1$, then the set $Q_{\varepsilon}(f) \backslash A(f)$ is non-empty.

If $f$ is not $\varepsilon$-regular, then for each $\ell \in \mathbb{N}$ there exists $n(\ell) \in \mathbb{N}$ such that

$$
\mu_{\varepsilon}^{n+\ell}(R)<M^{n}(R), \text { for } n \geq n(\ell) .
$$

Next, by Theorem [3.1, with $a_{n}=\mu_{\varepsilon}^{n}(R), n \in \mathbb{N}$, there exists a point $\zeta$ and a sequence $\left(n_{j}\right)$ with $n_{j} \rightarrow \infty$ as $j \rightarrow \infty$, such that

$$
\left|f^{n}(\zeta)\right| \geq \mu_{\varepsilon}^{n}(R), \text { for } n \in \mathbb{N},
$$

and

$$
\left|f^{n_{j}}(\zeta)\right| \leq M^{2}\left(\mu_{\varepsilon}^{n_{j}}(R)\right), \text { for } j \in \mathbb{N}
$$


It follows from (3.4) that $\zeta \in Q_{\varepsilon}(f)$. Also, (3.5) and (3.3) together imply that, for each $\ell \in \mathbb{N}$, we have

$$
\left|f^{\left(n_{j}-\ell+2\right)+\ell-2}(\zeta)\right|=\left|f^{n_{j}}(\zeta)\right| \leq M^{2}\left(\mu_{\varepsilon}^{n_{j}}(R)\right)<M^{2}\left(M^{n_{j}-\ell}(R)\right)=M^{n_{j}-\ell+2}(R),
$$

for sufficiently large values of $j$. Hence $\zeta \notin A(f)$, so $Q_{\varepsilon}(f) \backslash A(f) \neq \emptyset$, as required.

Part (b) of Theorem 1.2 follows easily from part (a). If $f$ is weakly regular, then $f$ is $\varepsilon$-regular for all $0<\varepsilon<1$, so $Q_{\varepsilon}(f)=A(f)$ for all $0<\varepsilon<1$, by part (a), and hence $Q(f)=A(f)$. In the other direction, if $Q(f)=A(f)$, then $Q_{\varepsilon}(f)=A(f)$ for all $0<\varepsilon<1$, by (1.2), so $f$ is $\varepsilon$-regular for all $0<\varepsilon<1$, by part (a), and hence $f$ is weakly regular.

Next we show that there exist transcendental entire functions $f$ such that $Q(f) \neq A(f)$.

Theorem 3.2. Let $0<\varepsilon<1$. There exists a transcendental entire function $f$ such that $Q_{\varepsilon}(f) \neq A(f)$, and hence $Q(f) \neq A(f)$.

We use the following lemma.

Lemma 3.3. Let

$$
f(z)=\prod_{m=1}^{\infty}\left(1-\frac{z}{r_{m}}\right) \text {, where } r_{m}>0 \text { for } m \in \mathbb{N} .
$$

Then there exist $\delta \in(0,1)$ and a sequence $\left(c_{m}\right)$, with $c_{m} \rightarrow 0$ as $m \rightarrow \infty$ and $0<c_{m}<1$ for $m \in \mathbb{N}$, such that, if $r_{m+1}>4 r_{m}$ for $m \in \mathbb{N}$, then

$$
\delta c_{m}|z|^{m}<|f(z)|<c_{m}|z|^{m}, \text { for } 2 r_{m}<|z|<\frac{1}{2} r_{m+1}, m \in \mathbb{N} \text {. }
$$

Proof. This follows easily by writing

$$
f(z)=\prod_{k=1}^{m} \frac{z}{r_{k}}\left(\frac{r_{k}}{z}-1\right) \prod_{k=m+1}^{\infty}\left(1-\frac{z}{r_{k}}\right) .
$$

Proof of Theorem 3.2. We now indicate how Lemma 3.3 can be used to show that, if $0<\varepsilon<1$ and $r_{m+1} / r_{m}$ is sufficiently large for each $m \in \mathbb{N}$, then the function $f$ given by $(\underline{3.6})$ is not $\varepsilon$-regular and hence $Q_{\varepsilon}(f) \neq A(f)$.

Let $\varepsilon^{\prime} \in(\varepsilon, 1)$. It follows from (3.7) that, for each $m \in \mathbb{N}$, if $r_{m+1} / r_{m}$ is sufficiently large, then there exists $R_{m}>r_{m}$ such that

$$
M(r)>r^{\varepsilon^{\prime} m} \text { and } \mu_{\varepsilon}(r)=M(r)^{\varepsilon}<r^{\varepsilon m}, \text { for } R_{m} \leq r<\frac{1}{2} r_{m+1} .
$$

Now fix $m \in \mathbb{N}$ and set $M_{m}(r)=r^{\varepsilon^{\prime} m}$ and $\mu_{\varepsilon, m}(r)=r^{\varepsilon m}$. Since $\varepsilon<\varepsilon^{\prime}$, there exists $N_{m} \in \mathbb{N}$ such that $(\varepsilon m)^{N_{m}+m}<\left(\varepsilon^{\prime} m\right)^{N_{m}}$ and hence, for $r>0$,

$$
\mu_{\varepsilon, m}^{N_{m}+m}(r)<M_{m}^{N_{m}}(r)
$$

So, if we choose $r_{m+1}$ sufficiently large to ensure that $M^{N_{m}}\left(R_{m}\right)<\frac{1}{2} r_{m+1}$, then it follows from (3.8) and (3.9), with $r=R_{m}$, that

$$
\mu_{\varepsilon}^{N_{m}+m}\left(R_{m}\right)<M^{N_{m}}\left(R_{m}\right) \text {, for } m \in \mathbb{N} .
$$

Now let $R$ be so large that $M(r)>r$, for $r \geq R$ and let $m \in \mathbb{N}$ be such that $R_{m}>M(R)>\mu_{\varepsilon}(R)$. Then there exists $N_{m}^{\prime} \in \mathbb{N}$ such that

$$
\mu_{\varepsilon}^{N_{m}^{\prime}}(R) \leq R_{m}<\mu_{\varepsilon}^{N_{m}^{\prime}+1}(R)
$$


It follows from (3.11) and (3.10) that

$$
\begin{aligned}
\mu_{\varepsilon}^{N_{m}+m+N_{m}^{\prime}}(R) & \leq \mu_{\varepsilon}^{N_{m}+m}\left(R_{m}\right)<M^{N_{m}}\left(R_{m}\right) \\
& <M^{N_{m}}\left(\mu_{\varepsilon}^{N_{m}^{\prime}+1}(R)\right)<M^{N_{m}+N_{m}^{\prime}+1}(R),
\end{aligned}
$$

and so, if $n$ is sufficiently large,

$$
\mu_{\varepsilon}^{n+m-1}(R)<M^{n}(R)
$$

Hence $f$ is not $\varepsilon$-regular, as required.

Remark In Section 6 we show by a different method that if $0<\varepsilon<\varepsilon^{\prime}<1$, then there exists a function that is $\varepsilon^{\prime}$-regular but not $\varepsilon$-regular, thus giving an alternative construction of a function for which $Q(f) \neq A(f)$.

\section{Regularity CONDITIONS}

Theorem 1.2 tells us that weak-regularity implies that $Q(f)=A(f)$. Unfortunately, weak-regularity is not straightforward to check directly. In this section we discuss several other regularity conditions and the relationships between these conditions and weak regularity. In particular, we discuss log-regularity which implies weak regularity and is often easy to check.

We say that a transcendental entire function $f$ is log-regular if there exists $c>0$ such that the function $\phi(t)=\log M\left(e^{t}\right)$ satisfies

$$
\frac{\phi^{\prime}(t)}{\phi(t)} \geq \frac{1+c}{t}, \text { for large } t \text {. }
$$

As mentioned earlier, the log-regularity condition was introduced by Anderson and Hinkkanen in [1] in relation to Baker's conjecture. The name log-regular was suggested by Aimo Hinkkanen in a private communication and used first by Sixsmith [21]. It was pointed out in [12, page 205] that this condition holds for all transcendental entire functions of finite order and positive lower order; recall that the $\operatorname{order} \rho$ and the lower order $\lambda$ of a transcendental entire function $f$ are:

$$
\rho=\limsup _{r \rightarrow \infty} \frac{\log \log M(r)}{\log r} \text { and } \lambda=\liminf _{r \rightarrow \infty} \frac{\log \log M(r)}{\log r} .
$$

The paper 21] gives many other classes of entire functions that are log-regular; for example, any composition of transcendental entire functions is log-regular if one of the functions in the composition is log-regular and any transcendental entire function $f$ is log-regular if and only if its derivative is log-regular.

There are also many transcendental entire functions which are not log-regular. For example, if $f$ has a multiply connected Fatou component, then $f$ is not logregular. This follows from the fact that such Fatou components contain large annuli within which the iterates of $f$ behave like the iterated maximum modulus of $f$ (see, for example, [6. Theorem 1.2 and Lemma 2.1]); combined with logregularity this fact would contradict the distortion theorem for iterates in a Fatou component [2, Lemma 7].

We now show that log-regularity implies weak-regularity. This result can also be deduced by combining results given in [15] and [16] (see Theorem 4.4part (d)) but we include a direct proof here for completeness. 
Theorem 4.1. Let $f$ be a transcendental entire function. If $f$ is log-regular, then $f$ is weakly regular.

Remark In Section 6] we give an example of an entire function which is weakly regular but not log-regular.

To prove Theorem 4.1, we first give a result on convex functions that leads to an alternative characterisation of log-regularity. This alternative characterisation is used again in Sections 5 and 6 of this paper to determine whether certain functions are or are not log-regular, and also in [21].

Theorem 4.2. Let $\phi$ be an increasing convex function defined on $\mathbb{R}$. The following are equivalent:

(a) there exist $t_{0}>0$ and $c>0$ such that

$$
\frac{\phi^{\prime}(t)}{\phi(t)} \geq \frac{1+c}{t}, \text { for } t \geq t_{0}
$$

(b) there exist $t_{0}>0$ and $c>0$ such that for all $k>1$ and $d=k^{c}$, we have

$$
\phi(k t) \geq k d \phi(t), \text { for } t \geq t_{0} ;
$$

(c) there exist $t_{1}>0, k>1$ and $d>1$, such that

$$
\phi(k t) \geq k d \phi(t), \text { for } t \geq t_{1} \text {. }
$$

Remark For definiteness, in the statement of this theorem $\phi^{\prime}(t)$ denotes the right derivative of $\phi$ at $t$.

To obtain our alternative characterisation of log-regularity we apply Theorem 4.2 with $\phi(t)=\log M\left(e^{t}\right)$.

Corollary 4.3. Let $f$ be a transcendental entire function. The following are equivalent:

(a) $f$ is log-regular;

(b) there exist $r_{0}>0$ and $c>0$ such that for all $k>1$ and $d=k^{c}$, we have

$$
M\left(r^{k}\right) \geq M(r)^{k d}, \text { for } r \geq r_{0} ;
$$

(c) there exist $r_{1}>0, k>1$ and $d>1$, such that

$$
M\left(r^{k}\right) \geq M(r)^{k d}, \text { for } r \geq r_{1} .
$$

Remarks 1. Corollary 4.3 part (b) shows that log-regularity represents a strengthening of the following version of Hadamard convexity, given in [15, Lemma 2.2]: for every transcendental entire function $f$ there exists $r(f)>0$ such that, for all $r \geq r(f)$ and all $k>1$,

$$
M\left(r^{k}\right) \geq M(r)^{k} .
$$

2. The condition in Corollary 4.3 part (c) is equivalent to a regularity condition used by Wang in [24], namely, that there exists $k>1$ such that

$$
\liminf _{r \rightarrow \infty} \frac{\log M\left(r^{k}, f\right)}{\log M(r, f)}>k
$$

Thus the latter condition is equivalent to log-regularity. 
Proof of Theorem 4.2. To prove that (a) implies (b), we note that, for $t \geq t_{0}$,

$$
\log \frac{\phi(k t)}{\phi(t)} \geq \int_{t}^{k t} \frac{\phi^{\prime}(u)}{\phi(u)} d u \geq \int_{t}^{k t} \frac{1+c}{u} d u=\log k^{1+c} .
$$

Next, it is clear that (b) implies (c).

To prove that (c) implies (a), suppose that $t \geq k t_{1}$. Since $\phi^{\prime}$ is increasing,

$$
t(1-1 / k) \phi^{\prime}(t) \geq \int_{t / k}^{t} \phi^{\prime}(u) d u=\phi(t)-\phi(t / k),
$$

so, using the fact that $\phi(t) \geq k d \phi(t / k)$, we have

$$
\frac{t \phi^{\prime}(t)}{\phi(t)} \geq \frac{1-\phi(t / k) / \phi(t)}{1-1 / k} \geq \frac{1-1 /(k d)}{1-1 / k}=1+c, \text { for } t \geq k t_{1},
$$

where $c>0$, as required.

Now we deduce Theorem 4.1

Proof of Theorem 4.1. Suppose that $f$ is log-regular with constant $c>0$ and let $\varepsilon$ be given with $0<\varepsilon<1$.

Let $R$ be so large that $M(r)>r$, for $r \geq R$. By Corollary 4.3 part (b), there exists $r_{0} \geq R$ such that for all $k>1$ and $d=k^{c}$, we have

$$
M\left(r^{k}\right) \geq M(r)^{k d}, \text { for } r \geq r_{0} .
$$

We apply this estimate with $d=1 / \varepsilon>1$ and $k=d^{1 / c}>1$. Then

$$
\mu_{\varepsilon}\left(r^{k}\right) \geq M(r)^{k}, \text { for } r \geq r_{0} .
$$

Thus, by induction,

$$
\mu_{\varepsilon}^{n}\left(r_{0}^{k}\right) \geq\left(M^{n}\left(r_{0}\right)\right)^{k} \geq M^{n}(R), \text { for } n \geq 0 .
$$

Hence $f$ is $\varepsilon$-regular.

We point out that there are other types of regularity conditions that are closely related to log-regularity and weak-regularity. These were introduced in [15] and [16] in relation to Baker's conjecture and the existence of a spider's web structure for the fast escaping set. We summarize the relationships between these conditions in the following theorem which shows that there are many examples of transcendental entire functions that are weakly regular.

Theorem 4.4. Let $f$ be a transcendental entire function and let $R>0$ be such that $M(r)>r$, for $r \geq R$. Then

(a) $f$ is $\varepsilon$-regular, where $0<\varepsilon<1$, if and only if there is a sequence $\left(r_{n}\right)$ such that

$$
r_{n} \geq M^{n}(R) \quad \text { and } \quad M\left(r_{n}\right) \geq r_{n+1}^{m}, \text { for } n \geq 0,
$$

where $m=1 / \varepsilon$;

(b) if $m>1$ and there exists a real function $\psi$ defined on $\left[r_{0}, \infty\right)$, where $r_{0}>0$, such that

$$
\psi(r) \geq r \text { and } M(\psi(r)) \geq(\psi(M(r)))^{m}, \text { for } r \geq r_{0},
$$

then there is a sequence $\left(r_{n}\right)$ such that (4.3) holds, so $f$ is $\varepsilon$-regular with $\varepsilon=1 / m$; 
(c) if $f$ is of finite order and there exist $n \in \mathbb{N}$ and $0<q<1$ such that

$$
M(r) \geq \exp ^{n+1}\left(\left(\log ^{n} r\right)^{q}\right), \text { for large } r,
$$

then, for each $m>1$, there is a real function $\psi$ such that (4.4) holds, so $f$ is weakly regular;

(d) if $f$ is log-regular, then for each $m>1$ there is a real function $\psi$ such that (4.4) holds, so $f$ is weakly regular.

Proof. To prove part (a) we first note that the condition (1.3) implies that (4.3) holds with $m=1 / \varepsilon$ and $r_{n}=\mu_{\varepsilon}^{n}(r), n \geq 0$. On the other hand, if (4.3) holds and $\varepsilon=1 / m$, then

$$
\mu_{\varepsilon}\left(r_{n}\right) \geq r_{n+1}, \text { for } n \geq 0
$$

SO

$$
\mu_{\varepsilon}^{n}\left(r_{0}\right) \geq r_{n} \geq M^{n}(R), \text { for } n \geq 0
$$

and hence (1.3) holds.

Part (b) follows from the fact that if $f$ satisfies the condition (4.4), for some value of $m$, then (4.3) holds for the same value of $m$, by taking $r_{n}=\psi\left(M^{n}(R)\right)$.

Part (c) is [15, Theorem 6]. There the function $f$ was assumed to have order less than $1 / 2$, but the proof actually required only finite order.

Part (d) was also proved in [15], by using a similar argument to the one given in the first part of the proof of Lemma 4.3. In [15, Section 7] we showed that if $f$ is log-regular, with constant $c>0$, then for all $m>1$ the statement (4.4) holds with $\psi(r)=r^{k}$, where $k=m^{1 / c}$.

Remarks 1. Theorem 4.4 part (a) shows that $\varepsilon$-regularity is equivalent to the type of regularity introduced in [16, Corollary 8.3 part (b)] with $m=1 / \varepsilon$.

2. A similar regularity condition to that in Theorem 4.4 part (b) played a key role in [21]. There the additional mild assumption was made that the function $\psi$ is increasing and the name $\psi$-regularity was used.

3. In Section 6 of this paper we use Theorem 4.4 part (c) to show that one of our examples is weakly regular.

Finally we mention two other regularity conditions. First, the condition

$$
\log M(2 r) \geq d \log M(r), \text { for large } r,
$$

where $d>1$, was mentioned in [3] in relation to a result on the packing dimension of $I(f) \cap J(f)$; see the remark after the proof of Theorem 5.1 of this paper. This regularity condition is easily seen to imply log-regularity. Next, the regularity condition

$$
\frac{\log M(2 r)}{\log M(r)} \rightarrow c \text { as } r \rightarrow \infty
$$

where $c \geq 1$, was used in 23 in relation to Baker's conjecture. This condition again implies $\log$-regularity in the case $c>1$, but it does not imply log-regularity in the case $c=1$. Indeed using an approximation method (see Lemma 6.3 of this paper) we can construct a transcendental entire function such that

$$
\log M(r) \sim \log r \log \log r \text { as } r \rightarrow \infty,
$$

and so $f$ satisfies (4.5) with $c=1$ but $f$ is not log-regular. 


\section{SufFiCiEnt CONDITIONS FOR LOG-REgularity}

First we prove the following sufficient condition for log-regularity, which is rather general; in particular, it does not require any hypothesis about the order of the function. Taken together with Theorem 4.1, this result implies Theorem 1.3 and hence Theorem 1.1.

Theorem 5.1. Let $f$ be a transcendental entire function such that, for some $r(f)>1$,

$$
m(r) \leq M(r)^{1-K / \log r}, \text { for } r \geq r(f),
$$

where $K=4 \log 4$. Then $f$ is log-regular.

In particular, if $f$ is in the class $\mathcal{B}$, then $f$ is log-regular.

We use the following result of Beurling [7, page 96], which also played a key role in [18]. Here, for any subset $E$ of $(0, \infty)$ we denote by $m_{\ell}(E)$ the logarithmic measure of $E$; that is,

$$
m_{\ell}(E)=\int_{E} \frac{d t}{t} .
$$

Lemma 5.2. Let $f$ be analytic in $\left\{z:|z|<r_{0}\right\}$, let $0 \leq r_{1}<r_{2}<r_{0}$, and put

$$
E=\left\{t \in\left(r_{1}, r_{2}\right): m(t) \leq \mu\right\} \text {, where } 0<\mu<M\left(r_{1}\right) \text {. }
$$

Then

$$
\log \frac{M\left(r_{2}\right)}{\mu}>c \exp \left(\frac{1}{2} m_{\ell}(E)\right) \log \frac{M\left(r_{1}\right)}{\mu},
$$

where $c=\pi /(4 \sqrt{2})$.

We remark that this result actually holds for all values of $\mu$ such that $0<\mu<$ $M\left(r_{2}\right)$ since for $M\left(r_{1}\right) \leq \mu<M\left(r_{2}\right)$ the right-hand side of (5.2) is negative or 0 .

Proof of Theorem 5.1. By Corollary 4.3 part (c), it is sufficient to show that there exist $k>1$ and $d>1$ such that

$$
\log M\left(r^{k}\right) \geq k d \log M(r), \text { for large } r .
$$

The idea of the proof is to use the estimate (5.1) together with Lemma 5.2 to show that, for sufficiently large $r$, the maximum modulus of $f$ must increase by a certain amount over each of a sequence of relatively short adjacent intervals of the form $[r, \lambda r]$, where $\lambda>1$, and then to combine these increases to obtain (5.3) for suitable values of $k$ and $d$.

First put

$$
\delta(r)=\frac{K}{\log r}, \quad r \geq r_{0}(f),
$$

where $r_{0}(f) \geq r(f)$ is so large that $\delta(r) \leq 1$ for $r \geq r_{0}(f)$. Now take $k>1$ and $\lambda$ such that $1<\lambda<r^{k-1}$ for $r \geq r_{0}(f)$, and apply Lemma 5.2 with

$$
r_{1}=s, \quad r_{2}=\lambda s, \quad \mu=M(\lambda s)^{1-\delta\left(r^{k}\right)}, \text { where } r \leq s<\lambda s \leq r^{k} .
$$

This gives

$$
\begin{aligned}
\log M(\lambda s)^{\delta\left(r^{k}\right)} & >c \exp \left(\frac{1}{2} \int_{s}^{\lambda s} \frac{d t}{t}\right) \log \frac{M(s)}{M(\lambda s)^{1-\delta\left(r^{k}\right)}} \\
& =c \lambda^{1 / 2}\left(\log M(s)-\left(1-\delta\left(r^{k}\right)\right) \log M(\lambda s)\right)
\end{aligned}
$$


SO

$$
\log M(\lambda s)>\frac{1}{\left(c \lambda^{1 / 2}\right)^{-1} \delta\left(r^{k}\right)+1-\delta\left(r^{k}\right)} \log M(s)
$$

If we now take

$$
\lambda=\frac{16}{\left(1-\delta\left(r^{k}\right)\right)^{2}},
$$

then $1<\lambda<r^{k-1}$ for $r \geq r_{1}(f, k) \geq r_{0}(f)$, say. Therefore, for $r \geq r_{1}(f, k)$ and $r \leq s<\lambda s \leq r^{k}$, we have

$$
\begin{aligned}
\log M(\lambda s) & >\frac{1}{(4 c)^{-1} \delta\left(r^{k}\right)\left(1-\delta\left(r^{k}\right)\right)+1-\delta\left(r^{k}\right)} \log M(s) \\
& >\frac{1}{1-a \delta\left(r^{k}\right)} \log M(s),
\end{aligned}
$$

where $a=1-(4 c)^{-1}$. Note that $1 / 2<a<1$, since $c=\pi /(4 \sqrt{2})>1 / 2$.

Then, for $r \geq r_{1}(f, k)$, we define $n=n(r, k) \in \mathbb{N}$ by the condition

$$
\lambda^{n} r \leq r^{k}<\lambda^{n+1} r
$$

and apply (5.5) to $s=\lambda^{m} r$, for $m=0,1, \ldots, n-1$. Combining these estimates gives

$$
\log M\left(r^{k}\right)>\left(\frac{1}{1-a \delta\left(r^{k}\right)}\right)^{n} \log M(r) .
$$

By the right-hand inequality in (5.6),

$$
\lambda^{n}>r^{(k-1) n /(n+1)},
$$

so (5.7) gives

$$
\log M\left(r^{k}\right)>\left(\lambda^{1 / 2}\right)^{n p(r)} \log M(r)>\left(r^{(k-1) / 2}\right)^{p(r) n /((n+1)} \log M(r),
$$

where

$$
p(r)=\frac{\log \left(1 /\left(1-a \delta\left(r^{k}\right)\right)\right)}{\log \lambda^{1 / 2}}=\frac{\log \left(1 /\left(1-a \delta\left(r^{k}\right)\right)\right)}{\log \left(4 /\left(1-\delta\left(r^{k}\right)\right)\right)} \geq \frac{a \delta\left(r^{k}\right)}{\log \left(4 /\left(1-\delta\left(r^{k}\right)\right)\right)},
$$

by (5.4).

Thus to deduce (5.3) from (5.8), it is sufficient to choose $k>1, d>1$ so that for sufficiently large $r$ we have

$$
\left(\frac{k-1}{2}\right)\left(\frac{n}{n+1}\right)\left(\frac{a \delta\left(r^{k}\right)}{\log \left(4 /\left(1-\delta\left(r^{k}\right)\right)\right)}\right) \log r \geq \log k d
$$

that is, since $\delta(r)=K / \log r$,

$$
\left(\frac{n}{n+1}\right)\left(\frac{2 a K}{4 \log \left(4 /\left(1-\delta\left(r^{k}\right)\right)\right)}\right) \geq\left(\frac{k}{k-1}\right) \log k d .
$$

To do this we first note that, for each fixed $k>1$,

$$
\delta\left(r^{k}\right) \rightarrow 0 \text { as } r \rightarrow \infty
$$

so, by (5.4) and (5.6),

$$
n=n(r, k) \rightarrow \infty \text { as } r \rightarrow \infty \text {. }
$$


Take $b=\frac{1}{2}(1+2 a)$ and note that $1<b<2 a$, because $a>1 / 2$. Since

$$
k \mapsto \frac{k \log k}{k-1} \text { is strictly increasing on }(1, \infty) \text {, with } \lim _{k \rightarrow 1} \frac{k \log k}{k-1}=1,
$$

and $b>1$, we can choose absolute constants $k>1$ and $d>1$ such that

$$
b>\left(\frac{k}{k-1}\right) \log k d .
$$

Thus, by (5.10) and (5.11), and the facts that $K=4 \log 4$ and $b<2 a$, we can choose $r_{2}(f) \geq r_{1}(f, k)$ such that (5.9) holds for all $r \geq r_{2}(f)$ with these values of $k$ and $d$. This completes the proof of Theorem 5.1.

Remark A similar condition to (5.1) appears in a recent paper of Bergweiler [3]. The main result of [3] is that if $f$ is a transcendental entire function with no multiply connected Fatou components and

$$
\frac{\log \log M(r)}{\log \log r} \rightarrow \infty \text { as } r \rightarrow \infty,
$$

then the packing dimension of $I(f) \cap J(f)=2$. Also, [3, Corollary 4.1] states that if

$$
m(r) \leq M(r)^{1-K(r) / \log r}, \text { for large } r,
$$

where $K(r) \rightarrow \infty$ as $r \rightarrow \infty$, then $f$ has no multiply connected Fatou components and (5.12) holds.

The assertion that (5.13) implies that $f$ has no multiply connected Fatou components is justified in [3] by using results from either [25] or [6], and the assertion that (5.13) implies (5.12) is deduced from a result of Fenton [11]. We remark that this latter implication can also be deduced by using the argument from the proof of Theorem 5.1 above. Briefly, (5.3) implies that

$$
\liminf _{r \rightarrow \infty} \frac{\log \log M(r)}{\log \log r} \geq \frac{\log k d}{\log k},
$$

and if $k=2$, say, while the constant $K$ in Theorem 5.1 is large, then (5.9) can be satisfied by a correspondingly large value of $d$. See also the related discussion of the consequences of log-regularity in [12, page 204].

We conclude this section by pointing out that Lemma 5.2 can be used to deduce other sufficient conditions for log-regularity, such as the following, in which the restriction on the minimum modulus holds only on a sufficiently large set.

Theorem 5.3. Let $f$ be a transcendental entire function. Suppose that there exist $\alpha, \beta, k$, with $0<\alpha<\beta<1, k>1$ and $r(f)>0$ such that the set

$$
F_{r}=\left\{\rho \in\left(r, r^{k}\right): m(\rho) \leq M\left(r^{k}\right)^{\alpha / k}\right\}, \quad r>0,
$$

satisfies

$$
m_{\ell}\left(F_{r}\right) \geq 2 \log \left(\frac{k-\alpha}{c(\beta-\alpha)}\right), \text { for } r \geq r(f),
$$

where $c=\pi /(4 \sqrt{2})$ is the constant in Lemma 5.2. Then $f$ is log-regular. 
Proof. Let $d=1 / \beta$. By Corollary 4.3 part (c), it is sufficient to show that

$$
\log M\left(r^{k}\right) \geq k d \log M(r)=\frac{k}{\beta} \log M(r), \text { for } r \geq r(f) .
$$

To do this we apply Lemma 5.2 with

$$
r_{1}=r, r_{2}=r^{k} \text { and } \mu=M\left(r^{k}\right)^{\alpha / k}
$$

where $r \geq r(f)$, which gives

$$
(1-\alpha / k) \log M\left(r^{k}\right)>\left(\log M(r)-(\alpha / k) \log M\left(r^{k}\right)\right) c \exp \left(m_{\ell}\left(F_{r}\right) / 2\right) ;
$$

that is,

$$
\left(1-\frac{\alpha}{k}+\frac{c \alpha}{k} \exp \left(m_{\ell}\left(F_{r}\right) / 2\right)\right) \log M\left(r^{k}\right)>c \exp \left(m_{\ell}\left(F_{r}\right) / 2\right) \log M(r) .
$$

Thus (5.14) holds if

$$
1-\frac{\alpha}{k}+\frac{c \alpha}{k} \exp \left(m_{\ell}\left(F_{r}\right) / 2\right) \leq\left(\frac{\beta}{k}\right) c \exp \left(m_{\ell}\left(F_{r}\right) / 2\right),
$$

and this is equivalent to

$$
m_{\ell}\left(F_{r}\right) \geq 2 \log \left(\frac{k-\alpha}{c(\beta-\alpha)}\right), \text { for } r \geq r(f),
$$

as required.

\section{EXAmples}

In this section we construct examples to show that log-regularity, $\varepsilon$-regularity and weak-regularity are not equivalent.

Example 6.1. There exists a transcendental entire function that is weakly regular but not log-regular.

Example 6.2. Suppose that $0<a<b<1$. Then there exists a transcendental entire function $f$ such that

(a) for all $\varepsilon \in[b, 1), f$ is $\varepsilon$-regular;

(b) for all $\varepsilon \in(0, a], f$ is not $\varepsilon$-regular and hence $Q_{\varepsilon}(f) \neq A(f)$.

It follows that $f$ is not weakly regular and $Q(f) \neq A(f)$.

We construct both examples using the following result of Clunie and Kovari [8].

Lemma 6.3. Let $\Phi$ be a convex increasing function on $\mathbb{R}$ such that $\Phi(t) \neq O(t)$ as $t \rightarrow \infty$. Then there exists a transcendental entire function $f$ such that

$$
\log M\left(e^{t}, f\right) \sim \Phi(t) \text { as } t \rightarrow \infty .
$$

Often we show that a function is $\varepsilon$-regular or weakly regular by first showing that it is log-regular. For these two examples, however, this method is not available and instead we use Theorem 4.4 part (c) in Example 6.1 and a direct construction in Example 6.2. 
Proof of Example 6.1. The idea is to start by constructing a 'model' function for $\log M\left(e^{t}, f\right)$, denoted by $\Phi(t)$, which is convex and satisfies the hypotheses of Theorem 4.4 part (c) but does not satisfy the log-regularity condition, and then apply Lemma 6.3 to $\Phi$ to obtain an entire function $f$ which also satisfies the hypotheses of Theorem 4.4 part (c), and so is weakly regular, but is not log-regular.

First, we define

$$
\mu(t)=\exp \left(t^{1 / 2}\right), \quad t \geq 0 .
$$

Then take $t_{0}>1$ so large that $\exp \left(\frac{3}{4} t^{1 / 2}\right)>t$ for $t \geq t_{0}$, and define

$$
t_{n}=\mu^{n}\left(t_{0}\right) \text { and } k_{n}=t_{n+1}^{1 / 4}, \quad n \geq 0 .
$$

Since $t_{n} \geq t_{0}, t_{n+1}^{3 / 4}>t_{n}$ and $k_{n}>1$, for $n \geq 0$, we have

$$
t_{n+1}>k_{n} t_{n}>t_{n}, \text { for } n \geq 0 .
$$

We now define

$$
\Phi(t)= \begin{cases}\mu_{n}(t), & t \in\left[t_{n+1} / k_{n}, t_{n+1}\right], n \geq 0, \\ \mu(t), & \text { otherwise }\end{cases}
$$

where $\mu_{n}(t)$ denotes the linear function such that $\mu_{n}(t)=\mu(t)$ for $t=t_{n+1} / k_{n}$ and $t=t_{n+1}$. Note that the intervals $\left[t_{n+1} / k_{n}, t_{n+1}\right]$ do not overlap, by (6.1). We claim that this function $\Phi$ has the following properties:

(a) $\Phi$ is convex;

(b) $\Phi$ satisfies

$$
\lim _{t \rightarrow \infty} \frac{\log \Phi(t)}{t}=0
$$

(c) for any $k>1$, we have

$$
\frac{\Phi\left(t_{n+1}\right)}{\Phi\left(t_{n+1} / k\right)} \rightarrow k \text { as } n \rightarrow \infty
$$

Property (a) is clear since the function $\mu$ is convex. To prove property (b), note that

$$
\limsup _{t \rightarrow \infty} \frac{\log \mu(t)}{t}=\limsup _{t \rightarrow \infty} t^{-1 / 2}=0
$$

and that

$$
\max \left\{\frac{\log \Phi(t)}{t}: t \in\left[t_{n+1} / k_{n}, t_{n+1}\right]\right\} \leq \frac{\log \mu\left(t_{n+1}\right)}{t_{n+1} / k_{n}}=\frac{1}{t_{n+1}^{1 / 4}} \rightarrow 0 \text { as } n \rightarrow \infty .
$$

Finally, property (c) holds because if $n$ is so large that $k_{n}>k$, then

$$
\begin{aligned}
\frac{\Phi\left(t_{n+1}\right)}{\Phi\left(t_{n+1} / k\right)} & =\frac{\mu\left(t_{n+1}\right)\left(t_{n+1}-t_{n+1} / k_{n}\right)}{\mu\left(t_{n+1} / k_{n}\right)\left(t_{n+1}-t_{n+1} / k\right)+\mu\left(t_{n+1}\right)\left(t_{n+1} / k-t_{n+1} / k_{n}\right)} \\
& <\frac{\mu\left(t_{n+1}\right)\left(t_{n+1}-t_{n+1} / k_{n}\right)}{\mu\left(t_{n+1}\right)\left(t_{n+1} / k-t_{n+1} / k_{n}\right)} \\
& =\frac{1-1 / k_{n}}{1 / k-1 / k_{n}} \rightarrow k \text { as } n \rightarrow \infty,
\end{aligned}
$$

as required. 
Now we apply Lemma 6.3 to $\Phi$ to give a transcendental entire function $f$ such that

$$
\log M\left(e^{t}, f\right)=\Phi(t)(1+\varepsilon(t))
$$

where $\varepsilon(t) \rightarrow 0$ as $t \rightarrow \infty$. Then

$$
\frac{\log \log M\left(e^{t}, f\right)}{t}=\frac{\log \Phi(t)}{t}+\frac{O(\varepsilon(t))}{t} \rightarrow 0 \text { as } t \rightarrow \infty
$$

by property (b), so $f$ has order 0 .

Also,

$$
\log M\left(e^{t}, f\right) \geq \frac{1}{2} \Phi(t) \geq \frac{1}{2} \exp \left(t^{1 / 2}\right) \geq \exp \left(t^{1 / 4}\right), \text { for large } t,
$$

so $f$ satisfies the hypotheses of Theorem 4.4 part (c), with $p=1$ and $q=1 / 4$. Hence $f$ is weakly regular.

Finally, by (6.2) and property (c), we have, for any $k>1$,

$$
\frac{\log M\left(e^{t_{n+1}}\right)}{\log M\left(e^{t_{n+1} / k}\right)} \sim \frac{\Phi\left(t_{n+1}\right)}{\Phi\left(t_{n+1} / k\right)} \rightarrow k \text { as } n \rightarrow \infty .
$$

Thus, if $r_{n}=e^{t_{n}}, n \in \mathbb{N}$, then

$$
M\left(r_{n+1}, f\right)=M\left(r_{n+1}^{1 / k}\right)^{k+o(1)} \text { as } n \rightarrow \infty,
$$

so $f$ is not log-regular.

Proof of Example 6.2. Recall that $0<a<b<1$. Once again the idea is to start by constructing a convex 'model' function $\Phi(t)$ for $\log M\left(e^{t}, f\right)$. In this case, $\Phi$ is constructed to be $\beta$-regular but not $\alpha$-regular, for some $\alpha$, $\beta$ with $a<\alpha<\beta<b$. Then we apply Lemma 6.3 to $\Phi$ to obtain an entire function $f$ which is $b$-regular but not $a$-regular.

First take $c \in(a, b)$ and construct a strictly increasing sequence $\left(t_{n}\right)$ as follows:

$$
t_{0}=1, \quad t_{1}=2, \frac{t_{n+2}}{t_{n+1}}=\left(\frac{1}{c}\right) \frac{t_{n+1}}{t_{n}}, n \geq 0 .
$$

Then let $\Phi$ be the function that satisfies

$$
\Phi(0)=0, \quad \Phi\left(t_{n}\right)=t_{n+1}, \quad n \geq 0,
$$

and is linear on $\left(-\infty, t_{0}\right]$ and on each interval $\left[t_{n}, t_{n+1}\right], n \geq 0$.

Next, for $0<s<1$, we let $\Psi_{s}(t)=s \Phi(t), t \in \mathbb{R}$, and we estimate the size of $\Psi_{s}(t)$ for $t \in\left[t_{n}, t_{n+1}\right]$. A general point $t \in\left[t_{n}, t_{n+1}\right]$ is of the form

$$
t=(1-\lambda) t_{n}+\lambda t_{n+1}, \text { where } 0 \leq \lambda \leq 1,
$$

and, by (6.4),

$$
\Psi_{s}(t)=s(1-\lambda) t_{n+1}+s \lambda t_{n+2} .
$$

By (6.5),

$$
\frac{t}{t_{n}}=1-\lambda+\lambda \frac{t_{n+1}}{t_{n}}
$$


and hence, by (6.6) and (6.4),

$$
\begin{aligned}
\frac{\Psi_{s}(t)}{t_{n+1}} & =s(1-\lambda)+s \lambda \frac{t_{n+2}}{t_{n+1}} \\
& =s(1-\lambda)+\left(\frac{s \lambda}{c}\right) \frac{t_{n+1}}{t_{n}} \\
& =s(1-\lambda)+\frac{s}{c}\left(\frac{t}{t_{n}}-(1-\lambda)\right) \\
& =\left(\frac{s}{c}\right) \frac{t}{t_{n}}-(1-\lambda) s\left(\frac{1-c}{c}\right) .
\end{aligned}
$$

Hence, for all $t \in\left[t_{n}, t_{n+1}\right]$ and $s \in(0,1)$, we have

$$
\frac{\Psi_{s}(t)}{t_{n+1}} \geq\left(\frac{s}{c}\right) \frac{t}{t_{n}}-\left(\frac{1-c}{c}\right)
$$

and

$$
\frac{\Psi_{s}(t)}{t_{n+1}} \leq\left(\frac{s}{c}\right) \frac{t}{t_{n}}
$$

To prove part (a) it is sufficient to prove that $f$ is $b$-regular. Put $\beta=\frac{1}{2}(c+b)$, so $c<\beta<b$. Then, by (6.7) with $s=\beta$ and $t \in\left[t_{n}, t_{n+1}\right]$, we have

$$
\frac{\Psi_{\beta}(t)}{t_{n+1}} \geq\left(\frac{\beta}{c}\right) \frac{t}{t_{n}}-\left(\frac{1-c}{c}\right) \geq\left(\frac{\beta+c}{2 c}\right) \frac{t}{t_{n}}>\frac{t}{t_{n}},
$$

provided that

$$
\frac{t}{t_{n}} \geq \frac{2(1-c)}{\beta-c}=\frac{4(1-c)}{b-c} .
$$

Thus if $N$ is so large that

$$
\frac{t_{N+1}}{t_{N}}>\frac{4(1-c)}{b-c}
$$

and $t \in\left[(4(1-c) /(b-c)) t_{N}, t_{N+1}\right]$, then, by repeated application of (6.9)), we obtain

$$
\frac{\Psi_{\beta}^{n}(t)}{t_{N+n}} \geq\left(\frac{\beta+c}{2 c}\right)^{n} \frac{t}{t_{N}}, \text { for } n \in \mathbb{N} .
$$

In particular, if $t \in\left[(4(1-c) /(b-c)) t_{N}, t_{N+1}\right]$, then

$$
\Psi_{\beta}^{n}(t) \geq t_{N+n}, \text { for } n \in \mathbb{N} \text {. }
$$

Since $0<\beta / b<1$, we can apply Lemma 6.3 to obtain a transcendental entire function $f$ and $N \in \mathbb{N}$ such that

$$
\left(\frac{\beta}{b}\right) \Phi(t) \leq \log M\left(e^{t}, f\right) \leq \Phi(t), \text { for } t \geq t_{N},
$$

and also such that (6.10) holds, and hence (6.11).

For $0<\varepsilon<1$, we put

$$
\mu_{\varepsilon}(r)=M(r, f)^{\varepsilon} \quad \text { and } \quad \psi_{\varepsilon}(t)=\log \mu_{\varepsilon}\left(e^{t}\right)=\log M\left(e^{t}, f\right)^{\varepsilon} .
$$

Then, by (6.12),

$$
\psi_{b}(t) \geq \beta \Phi(t)=\Psi_{\beta}(t), \text { for } t \geq t_{N} .
$$


Therefore, if $t \in\left[(4(1-d) /(b-d)) t_{N}, t_{N+1}\right]$, then, by (6.11) and (6.12) again,

$$
\psi_{b}^{n}(t) \geq \Psi_{\beta}^{n}(t) \geq t_{N+n}=\Phi^{n}\left(t_{N}\right) \geq \log M^{n}\left(e^{t_{N}}, f\right), \text { for } n \in \mathbb{N},
$$

SO

$$
\mu_{b}^{n}\left(e^{t}\right) \geq M^{n}\left(e^{t_{N}}, f\right), \text { for } n \in \mathbb{N} \text {. }
$$

Hence $f$ is $b$-regular.

To prove part (b) it is sufficient to show that $f$ is not $a$-regular. Put $\alpha=$ $\frac{1}{2}(a+c)$, so $a<\alpha<c$. Then, by (6.8) with $s=\alpha$ and $t \in\left[t_{n}, t_{n+1}\right]$, we have

$$
\frac{\Psi_{\alpha}(t)}{t_{n+1}} \leq\left(\frac{\alpha}{c}\right) \frac{t}{t_{n}}, \text { for } n \in \mathbb{N}
$$

We deduce, by (6.12), (6.13) and (6.14), that if $t \in\left[t_{n}, t_{n+1}\right]$, then

$$
\frac{\log \mu_{a}\left(e^{t}\right)}{t_{n+1}}=\frac{a \log M\left(e^{t}\right)}{t_{n+1}} \leq \frac{a \Phi(t)}{t_{n+1}}=\left(\frac{a}{\alpha}\right) \frac{\Psi_{\alpha}(t)}{t_{n+1}} \leq\left(\frac{a}{c}\right) \frac{t}{t_{n}}, \text { for } n \in \mathbb{N} \text {. }
$$

Since $0<a / c<1$, we deduce that for any $n \in \mathbb{N}$ and $t \in\left(t_{n}, t_{n+1}\right]$ there exists $k \in \mathbb{N}$ such that

$$
\frac{\log \mu_{a}^{k}\left(e^{t}\right)}{t_{n+k}} \leq\left(\frac{a}{c}\right)^{k} \frac{t}{t_{n}} \leq 1, \quad \text { so } \quad \log \mu_{a}^{k}\left(e^{t}\right) \leq t_{n+k}=\Phi^{k}\left(t_{n}\right) .
$$

By applying this result repeatedly we see that for any $n \in \mathbb{N}, t \in\left[t_{n}, t_{n+1}\right]$ and $\ell \in \mathbb{N}$ there exists $k \in \mathbb{N}$ such that

$$
\log \mu_{a}^{k}\left(e^{t}\right) \leq t_{n+k-\ell}
$$

Now let

$$
\phi(t)=\log M\left(e^{t}, f\right), \quad t \in \mathbb{R} .
$$

Then, by (6.12), we have

$$
\Phi(t) \geq \phi(t) \geq\left(\frac{\beta}{b}\right) \Phi(t)>\beta \Phi(t)=\Psi_{\beta}(t), \text { for } t \geq t_{N} .
$$

Thus if we put

$$
T_{n}=\phi^{n}\left(t_{N}\right), \quad n \geq 0,
$$

then $\Phi^{n}\left(t_{N}\right) \geq T_{n} \geq \Psi_{\beta}^{n}\left(t_{N}\right)$, for $n \geq 0$, so it follows by (6.11) that

$$
T_{n} \in\left(t_{N+n-1}, t_{N+n}\right], \text { for } n \geq 0 .
$$

Thus, for any $n \geq N$ and $t \in\left[t_{n}, t_{n+1}\right]$ we can take $\ell=n+1-N$ in (6.15) and deduce that there exists $k \in \mathbb{N}$ such that

$$
\log \mu_{a}^{k}\left(e^{t}\right) \leq t_{N+k-1}<T_{k},
$$

by (6.18). Hence, for any sufficiently large $t$, there exists $k \in \mathbb{N}$ such that

$$
\mu_{a}^{k}\left(e^{t}\right)<\exp \left(T_{k}\right)=M^{k}\left(e^{T_{N}}\right),
$$

by (6.16) and (6.17), and it follows that $f$ is not $a$-regular. 


\section{REFERENCES}

[1] J.M. Anderson and A. Hinkkanen, Unbounded domains of normality, Proc. Amer. Math. Soc., 126 (1998), 3243-3252.

[2] W. Bergweiler, Iteration of meromorphic functions, Bull. Amer. Math. Soc., 29 (1993), $151-188$.

[3] W. Bergweiler, On the packing dimension of the Julia set and the escaping set of an entire function, Israel J. Math., 129 (2012), 449-472.

[4] W. Bergweiler and A. Hinkkanen, On semiconjugation of entire functions, Math. Proc. Camb. Phil. Soc., 126 (1999), 565-574.

[5] W. Bergweiler, B. Karpińska and G. M. Stallard, The growth rate of an entire function and the Hausdorff dimension of its Julia set, J. London Math. Soc., 80 (2009), 680-698.

[6] W. Bergweiler, P.J. Rippon and G.M. Stallard, Multiply connected wandering domains of entire functions. To appear in Proc. London Math. Soc., arXiv: 1109.1794.

[7] A. Beurling, Étude sur un problème de majoration, Uppsala, 1933.

[8] J.G. Clunie and T. Kövari, On integral functions having prescribed asymptotic growth, II, Canad. J. Math., 20 (1968), 7-20.

[9] A.E. Eremenko, On the iteration of entire functions, Dynamical systems and ergodic theory, Banach Center Publications 23, Polish Scientific Publishers, Warsaw, 1989, 339-345.

[10] A.E. Eremenko and M.Yu. Lyubich, Dynamical properties of some classes of entire functions, Ann. Inst. Fourier, Grenoble, 42 (1992), 989-1020.

[11] P.C. Fenton, The infimum of small subharmonic functions, Proc. Amer. Math. Soc., 78 (1980), 43-47.

[12] A. Hinkkanen, Entire functions with bounded Fatou components, Transcendental dynamics and complex analysis, 187-216, Cambridge University Press, 2008.

[13] J. Peter, Hausdorff measure of escaping and Julia sets for bounded type functions of finite order. To appear in Ergodic Theory and Dynam. Systems, arXiv: 1102.4933.

[14] P.J. Rippon and G.M. Stallard, On questions of Fatou and Eremenko, Proc. Amer. Math. Soc., 133 (2005), 1119-1126.

[15] P.J. Rippon and G.M. Stallard, Functions of small growth with no unbounded Fatou components, Journal d'Analyse Math., 108 (2009), 61-86.

[16] P.J. Rippon and G.M. Stallard, Fast escaping points of entire functions, Proc. London Math. Soc., 105 (2012), 787-820.

[17] P.J. Rippon and G.M. Stallard, Baker's conjecture and Eremenko's conjecture for functions with negative zeros. To appear in Journal d'Analyse Math., arXiv: 1112.5103.

[18] P.J. Rippon and G.M. Stallard, A sharp growth condition for a fast escaping spider's web. Preprint. arXiv:1208.3371,

[19] P.J. Rippon and G.M. Stallard, Annular itineraries for entire functions. Preprint. arXiv: 1301.1328.

[20] G. Rottenfußer, J. Rückert, L. Rempe and D. Schleicher, Dynamic rays of bounded-type entire functions, Ann. of Math., 173 (2011), 77-125.

[21] D.J. Sixsmith, Examples of functions for which the escaping set is a spider's web, Math. Proc. Camb. Phil. Soc., 151 (2011), 551-571.

[22] D.J. Sixsmith, Simply connected fast escaping Fatou components, Comm. Pure Appl. Math., 8 (2012), 1029-1046.

[23] G.M. Stallard, The iteration of entire functions of small growth, Math. Proc. Cambridge Philos. Soc., 114 (1993), 43-55.

[24] Y. Wang, On the Fatou set of an entire function with gaps, Tohoku Math. J., 53 (2001), $163-170$.

[25] J.H. Zheng, On multiply-connected Fatou components in iteration of meromorphic functions, J. Math. Anal. Appl., 313 (2006), 24-37. 
Department of Mathematics and Statistics, The Open University, Walton Hall, Milton Keynes MK7 6AA, UK

E-mail address: p.j.rippon@open.ac.uk

Department of Mathematics and Statistics, The Open University, Walton Hall, Milton Keynes MK7 6AA, UK

E-mail address: g.m.stallard@open.ac.uk 